\title{
Malignant Melanoma Arising in Red Tattoo Ink
}

\author{
Cormac Weekes Joyce ${ }^{1,2}$, Gerald Duff ${ }^{1}$, Dermot McKenna ${ }^{3}$, Padraic James Regan ${ }^{1,2}$ \\ ${ }^{1}$ Department of Plastic Surgery, University Hospital Galway, Galway; ${ }^{2}$ National University of Ireland, Gaillimh; ${ }^{3}$ Department of \\ Dermatology, Sligo Regional Hospital, Sligo, Ireland
}

We report the case of a 33-year-old male who presented with a malignant melanoma on his anterior chest wall. The lesion was only found in the red ink pigment of the tattoo, as were several in-transit dermal metastases. Possible explanations include a pre-existing lesion which was seeded with red ink or the possibility of the red ink causing an inflammatory reaction leading to malignant transformation. This is the first reported case of a melanoma developing in the red ink pigment of a multi-colored tattoo.

Keywords: Melanoma / Skin / Cutaneous
Correspondence: Cormac W Joyce Department of Plastic Surgery, University Hospital Galway, Newcastle Road, Galway, Ireland Tel: $+353-876815514$ Fax: +353-876815514 E-mail: cjoyce78@hotmail.com

No potential conflict of interest relevant to this article was reported.

Received: 7 Feb 2015 • Revised: 3 Mar 2015• Accepted: 23 Apr 2015

pISSN: 2234-6163 • elSSN: 2234-6171 • http://dx.doi.org/10.5999/aps.2015.42.4.475 • Arch Plast Surg 2015;42:475-477

\section{INTRODUCTION}

Skin malignancies arising in tattoos have been reported in the literature but are exceedingly rare. The process of tattooing involves the integration of metallic salts and organic dyes into the dermis. After injection, the dye can be found between the epidermis and upper dermis and is phogocytosed by immune cells. Later on, granulation occurs in the dermis and new connective tissue is created and so the dye becomes trapped within fibroblasts. This results in an increased concentration of dye just below the junction of the epidermis and dermis. Over time, this pigment migrates deeper within the dermis.

\section{CASE}

A 33-year-old man was referred by the Dermatology service with four nodules arising in a tattoo on his left anterior chest wall (Fig. 1). The tattoo had been carried out in the United Kingdom three years prior to the onset of the nodule formation. An initial nodule arose in the central left portion of his chest and this was followed several months later by three more nodules, all-arising within red parts of the multicolour tattoo. The patient was uncertain if there had been a mole on his left chest prior to getting the tattoo. He had no past medical history of any significance and he had worked for 10 years in the merchant navy yet claimed he had little sun exposure.

Excisional biopsies of these lesions revealed four melanomatous lesions, the maximum of which had a Breslow depth of 11 $\mathrm{mm}$. Pathological assessment of these lesions felt that they consisted of a primary tumour of $11 \mathrm{~mm}$ depth and three in-transit metastases. Microscopy of the primary lesion demonstrated sheets of highly atypical cells, which extended into the underlying dermis as atypical spindled and stellate cells with prominent perineural invasion. Focal epidermal ulceration was present along with a junctional component in areas. Tumour cells stained positive with S100, but negative for HMB45, Melan A and AE1/ AE3. It was felt that it was a nodular subtype melanoma with a Clark's level of IV with no features of regression. On clinical examination there was no cervical or axillary lymphadenopathy. A computed tomography (CT) thorax, abdomen and pelvis revealed some subcentimetre lymph nodes in the left axilla and jejunum. A subsequent positron emission tomography-CT did 


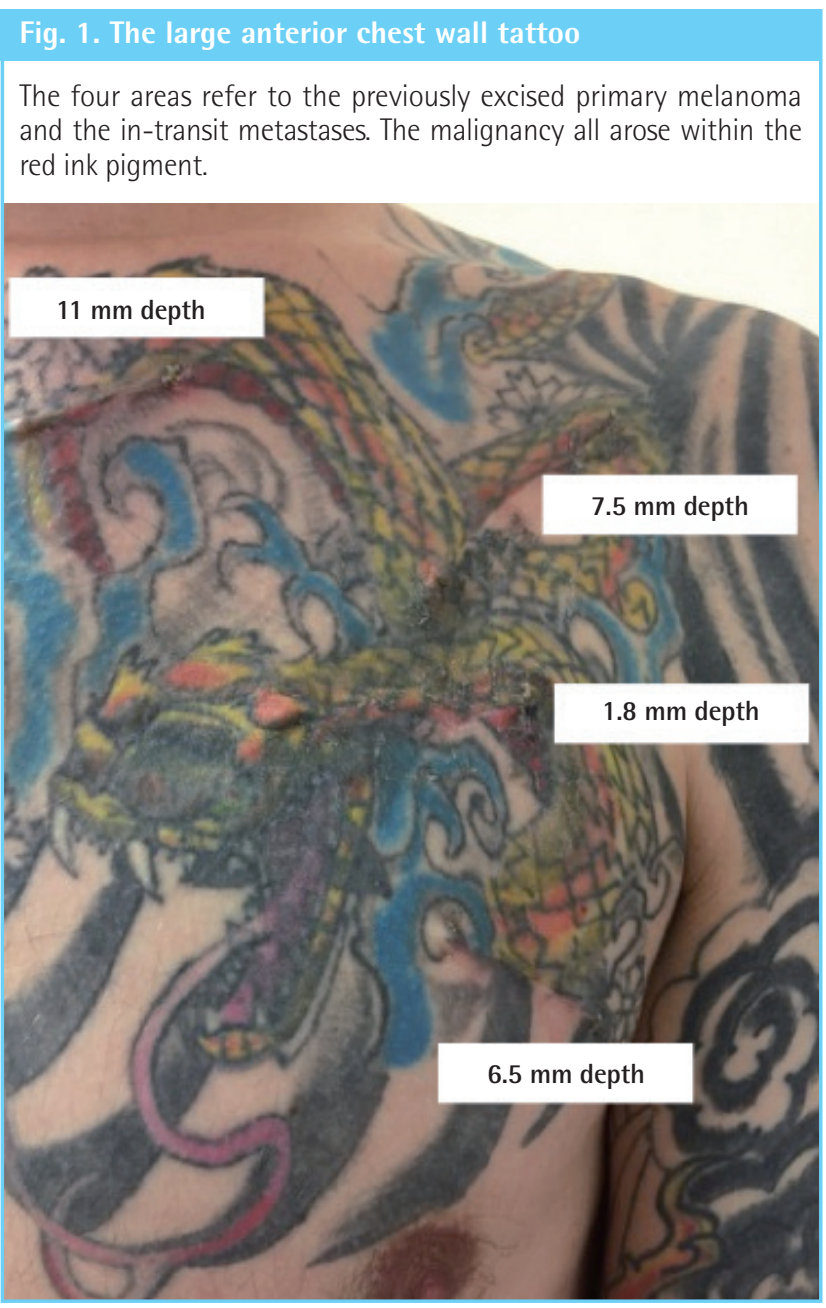

not show increased metabolic activity in these areas.

An en-bloc wide local excision of the nodules was carried out with a $2 \mathrm{~cm}$ margin down to pectoral fascia (Figs. 2, 3). A sentinel node biopsy was also performed which was positive. The chest defect was reconstructed with a split-thickness skin graft and he underwent an axillary lymph node clearance.

\section{DISCUSSION}

To our knowledge, this is the first reported case of a melanoma arising specifically in the red ink of a tattoo. They have been reported as arising in tattoos previously, but not within a particular ink pigment $[1,2]$. The pathogenesis of melanoma arising in a tattoo is unknown. Various dermatological conditions have been reported arising in tattoos including basal cell carcinomas [3], keratocanthomas and squamous cell carcinomas [4] yet there has been very little reports of melanoma developing in pre-existing tattoos [5]. Tattoos are not considered a risk factor for the development of melanoma. Several postulations have been credited with melanoma formation in tattoos including a
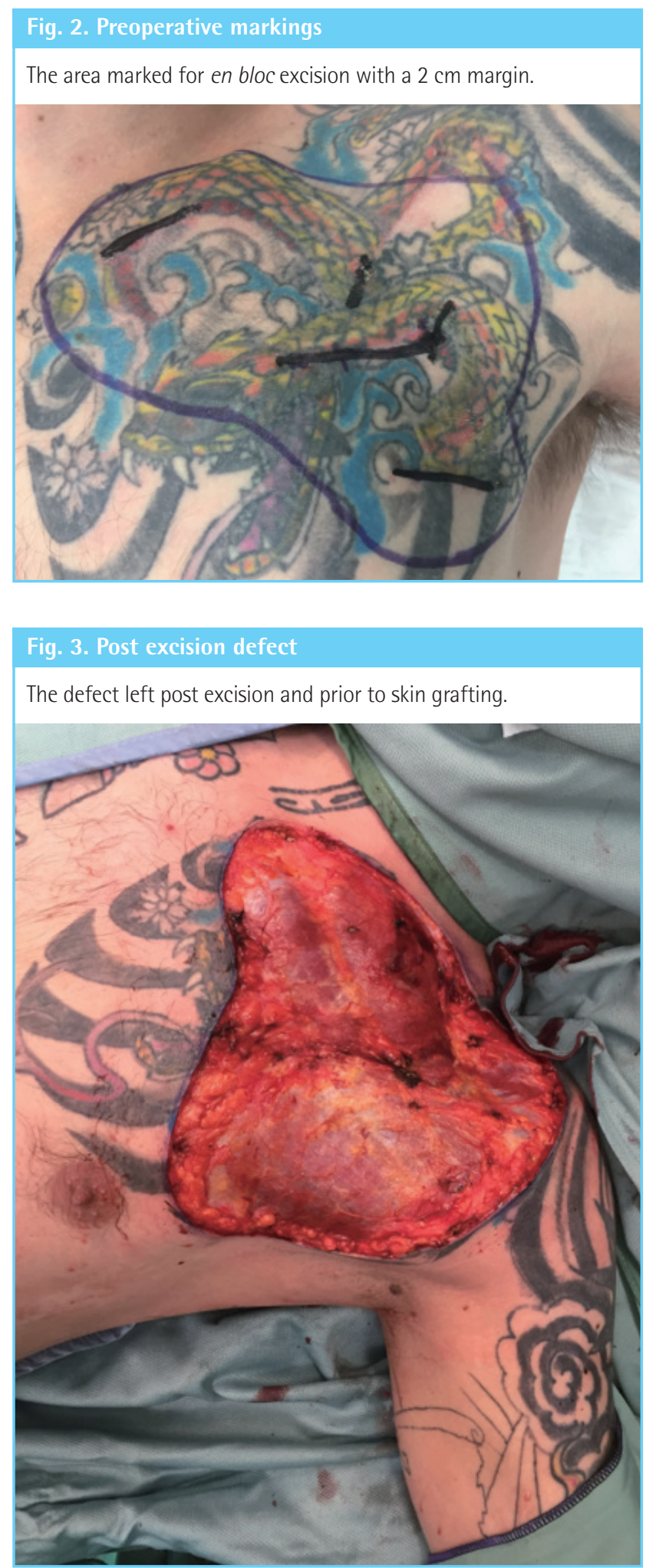

photo allergic effect, a chronic inflammatory reaction or trauma stimulating a malignant transformation. The previously reported melanomas arising in tattoos differ in their methods and materials used so it is difficult to ascertain a link between them. Several patients had radiotherapy to the area so that may have 
been a causative factor. Other patients had a pre-existing lesion and so the melanoma arising in the tattoo was likely coincidental. What makes our case so unique is that the melanoma and metastatic deposits were only found in the red parts of a large multi-coloured tattoo. A potential explanation is that our patient may have had a pre-existing melanoma and that the red ink was seeded with melanoma cells.

Tattoos are becoming increasingly popular in developing countries and the development of many different colours has allowed tattoos to become more intricate. The exact composition of tattoo ink is not internationally standardized and as a result it varies from studio to studio [6] and it is recognized that carcinogens can be found in many types of ink. In the past, dermatologic reactions to red tattoo ink was linked to mercury sulphide but this is no longer used in ink production [4]. However, reactions to red ink remain the most common of all the colours used in tattoos [7].

We report on an unusual case of a nodular malignant melanoma arising within the red pigment of a multicoloured tattoo. The pathogenesis of how it occurred is unknown but it may have been due to a pre-existing lesion that was seeded by the red ink or it may have arose as a malignant transformation secondary to the red tattoo ink.

\section{REFERENCES}

1. Nolan KA, Kling M, Birge M, et al. Melanoma arising in a tattoo: case report and review of the literature. Cutis 2013; 92:227-30.

2. Paradisi A, Capizzi R, De Simone C, et al. Malignant melanoma in a tattoo: case report and review of the literature. Melanoma Res 2006; 16:375-6.

3. Lee JS, Park J, Kim SM, et al. Basal cell carcinoma arising in a tattooed eyebrow. Ann Dermatol 2009;21:281-4.

4. Paprottka FJ, Bontikous S, Lohmeyer JA, et al. Squamouscell carcinoma arises in red parts of multicolored tattoo within months. Plast Reconstr Surg Glob Open 2014;2:e114.

5. Varga E, Korom I, Varga J, et al. Melanoma and melanocytic nevi in decorative tattoos: three case reports. J Cutan Pathol 2011;38:994-8.

6. Kluger N, Koljonen V. Tattoos, inks, and cancer. Lancet Oncol 2012;13:e161-8.

7. Mortimer NJ, Chave TA, Johnston GA. Red tattoo reactions. Clin Exp Dermatol 2003;28:508-10. 